\title{
Leucemias Agudas na Infância e Adolescência
}

\author{
Acute leukemia in childhood and adolescence
}

\section{I - LEUCEMIA LINFOBLÁSTICA AGUDA (LLA)}

\section{INTRODUÇÃOO}

Desde o ano de 1982, o Serviço de $\mathrm{H}$ ematologia do INCA vem utilizando 0 protocolo do G rupo Europeu Berlim-Frankfurt$M$ ünster (BFM ) para o tratamento da Leucemia Linfoblástica Aguda (LLA) da criança e do adolescente até 15 anos de idade. 0 btendo uma sobrevida livre de doença de $64 \%$, em mais de 6 mil dias, nos três grupos de risco, com os Protocolos BFM -81, BFM -83 e BFM 86, passou-se a adotar o Protocolo BFM -90. Este foi substituído pelo BFM -95, pronto se divulgaram os piores resultados observados no grupo de alto risco com o BFM -90.

Algumas modificações, entretanto, foram feitas pelo Serviço de H ematologia do IN CA no atual protocolo: Redução da dose de M ethotrexato (M TX) de $5 \mathrm{~g} / \mathrm{m}^{2}$ para $2 \mathrm{~g} / \mathrm{m}^{2}$, substituição da Vindesina pela Vincristina e redução da posologiada L-Asparaginasede 25.000 $\mathrm{U} / \mathrm{m}^{2}$ em infusão venosa de 6 horas para 10.000 $\mathrm{U} / \mathrm{m}^{2}$ por via intramuscular. Também, o Serviço incluiu a infiltração testicular ou do sistema nervoso central (SNC) e a faixa etária abaixo de 1 ano e igual ou mais de 10 anos como critérios de inclusão no grupo de alto risco.

Foi mantido o acompanhamento da dosagem do nível sérico de MTX, em todos os grupos de risco, e a utilização do Fator de Crescimento de Colônias (G-CSF) a partir do terceiro dia após o término de cada bloco terapêutico do grupo de alto risco.

Experimentalmente, visando a um maior índice de cura, além de um aprimoramento do diagnóstico e do estudo da LLA da criança e do adolescente, o serviço de hematologia do INCA vem participando do Grupo Brasileiro de Tratamento de Leucemia da Infância (GBT LI) - LLA/99.

\section{AVALIAÇÃO INICIAL \\ 1. CONFIRMAÇÃO DIAGNÓSTICA E CARACTERIZAÇÃO BIOLÓGICA}

É feito através do exame citológico do sangue periférico, da medula óssea e do líquor (líquido céfalo-raquidiano - LCR). 0 diagnóstico de LLA é estabelecido, quando $25 \%$ ou mais das células nucleadas da medula óssea são linfoblastos.

O s seguintes exames são procedidos:

- Citomorfologia e Citoquímica (PAS, Sudam Black e Fosfatase Ácida) do sangue periférico ou da medula óssea.

- Imunofenotipagem do sangue periférico ou da medula óssea.

- Citogenética convencional da medula óssea ou do sangue periférico.

N OTA: Exame de Biologia M olecular do sangue periférico ou da medula óssea tem sido procedido, servindo, posteriormente, como marcador da D oença Residual M ínima (DRM ).

\section{PROCEDIMENTOS DIAGNÓSTICOS NAS MANIFESTAÇÕES EXTRAMEDULARES}

- Exame físico, pesquisando as cadeias ganglionares, tamanho do fígado, baço e testículos e alterações neurológicas.

- Radiografia simples (RX) de tórax, com avaliação do mediastino.

- RX do local afetado, quando houver dor óssea, e Cintigrafia Ó ssea.

- Ultra-sonografia dos testículos, quando houver aumento testicular.

- Biópsia testicular, se houver dúvida quanto ao acometimento.

- Exame do fundo de olhos olho.

\section{GRUPOS DE RISCO}

\subsection{Grupo de risco básico:}

- Células leucêmicas abaixo de $1.000 / \mu l$ no sangue periférico, no Dia 8, após 7 dias de Prednisona e 1 injeção de M TX intratecal (IT). 
- Fator de Risco (FR)* <0,8 (todos os oito critérios devem estar preenchidos).

- Remissão completa no Dia 33 (M edula M 1).

- Ausência de translocação t(9;22) respectivamente recombinação $B C R / A B L$.

- Ausência de translocação $t(4 ; 11)$ respectivamente recombinação M LL/AF4.

- Idade $\geq 1$ ano e <10 anos.

- Ausência de imunologia T.

- Ausência de infiltração testicular ou do SN C.

3.2. Grupo de risco médio:

- Células leucêmicas abaixo de 1.000/ $\mu$ no sangue periférico no Dia 8, após 7 dias de Prednisona e 1 injeção de M TX IT.

- Remissão completa no Dia 33 (M edula M 1).

- Ausência de translocação t(9;22) respectivamente recombinação $B C R / A B L$.

- Ausência de translocação $t(4 ; 11)$ respectivamente recombinação M LL/AF4.

- $F R \geq 0,8$ e <1,2 (ver 3.1 e 3.3).

- Ausência de infiltração testicular ou do SN C.

\subsection{Grupo de risco alto:}

- Células leucêmicas $\geq$ de $1.000 / \mu l$ no sangue periférico, no Dia 8 após 7 dias de Prednisona e uma injeção de M TX IT.

- Ausência de remissão completa no D 33 (M edula M 2 ou M 3).

- Translocação $t(9 ; 22)$ respectivamente recombinação $B C R / A B L$.

- Translocação $t(4 ; 11)$ respectivamente recombinação M LL/AF4.

- $F R \geq 1,2$ (cada critério isolado classifica 0 paciente como Alto Risco).

- Infiltração testicular ou do SN C.

- Idade $<1$ ano e $\geq 10$ anos.

\section{TRATAMENTO}

\section{FUNDAMENTOS DA DOSAGEM}

- A dosagem dos citostáticos é feita de acordo com a superfície corpórea que deve ser determinada a cada mudança de fase de cada protocolo.

- Profilaxia da cistite: M esna - 1/3 da dose da Ciclofosfamida ou da I fosfamida, endovenoso, no momento 0, 4 e 8 hs após início da quimioterapia.

- Lactentes recebem, de acordo com a idade, uma dose reduzida de citostáticos: D o nascimento ao $6^{\circ}$ mês de vida: $2 / 3$ da dose calculada; do $7^{\circ}$ ao $12^{\circ}$ mês de vida: $3 / 4$ da dose calculada.

- Dosagem de M TX IT ou intra-ventricular de acordo com a idade:

\begin{tabular}{l|c} 
IDADE & DOSE \\
$<1$ ano & $6 \mathrm{mg}$ \\
\hline$\geq 1$ ano $<2$ anos & $8 \mathrm{mg}$ \\
\hline$\geq 2$ anos $<3$ anos & $10 \mathrm{mg}$ \\
\hline$\geq 3$ anos & $12 \mathrm{mg}$ \\
\hline
\end{tabular}

- D osagem do M ADIT (M ethotrexato/Ara-C/ D exametasona IT ):

\begin{tabular}{l|c|c|c} 
IDADE & MTX IT (mg) & ARA C IT (mg) & DEXA IT (mg) \\
$<1$ ano & 6 & 16 & $2 \mathrm{mg} / \mathrm{m}^{2}$ (máximo $\left.2 \mathrm{mg}\right)$ \\
\hline$\geq 1<2$ anos & 8 & 20 & $2 \mathrm{mg} / \mathrm{m}^{2}$ (máximo $\left.2 \mathrm{mg}\right)$ \\
\hline$\geq 2<3$ anos & 10 & 26 & $2 \mathrm{mg} / \mathrm{m}^{2}$ (máximo $\left.2 \mathrm{mg}\right)$ \\
\hline$\geq 3$ anos & 12 & 30 & $2 \mathrm{mg} / \mathrm{m}^{2}$ (máximo $\left.2 \mathrm{mg}\right)$ \\
\hline
\end{tabular}

PLANO GERAL DO PRODUTO

Grupo de Risco
Básico

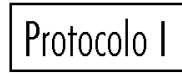

\section{Protocolo II}

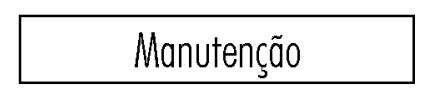

\section{Grupo de Risco Médio}
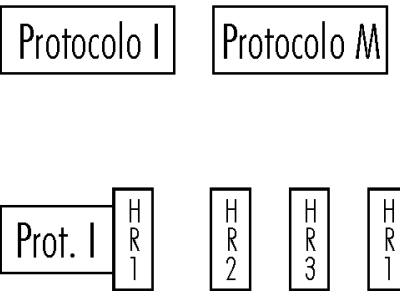

Protocolo II $126 y$
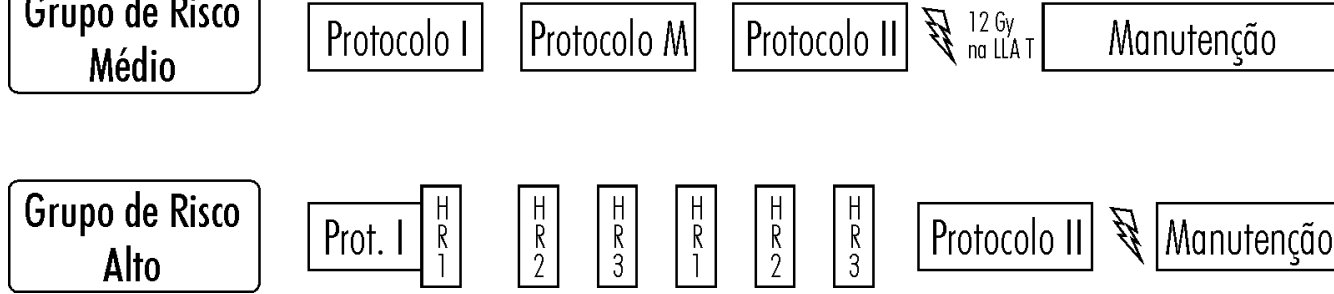

Protocolo II Manutenção

*Fator de Risco (FR) é baseado no número de blastos do sangue periférico ao diagnóstico e no tamanho do baço e do fígado, conformeProtocolos do Grupo Europeu BFM , anteriores ao Protocolo BFM 95. 


\section{INDUCC̃̃O - PROTOCOLO I}

Comum a todos os grupos de risco.

\begin{tabular}{l|c|c} 
PROTOCOLO I & DOSES & DIAS \\
Prednisona & $60 \mathrm{mg} / \mathrm{m}^{2} \mathrm{VO}$ & 1 a 28 \\
\hline Vincristina & $1,5 \mathrm{mg} / \mathrm{m}^{2} \mathrm{IV}$ & $8,15,22,29$ \\
\hline Daunorubicina & $30 \mathrm{mg} / \mathrm{m}^{2} \mathrm{IV}$ & $8,15,22,29$ \\
\hline Asparaginase & $5.000 \mathrm{U} / \mathrm{m}^{2} \mathrm{IM}$ & $12,15,18,21,24,27,30$ e 33 \\
\hline MTX IT, se SNC negativo & dose segundo idade & $1,12,33,45$ e 59 \\
\hline MTX IT, se SNC positivo & dose segundo idade & $1,12,18,27,33,45$ e 59 \\
\hline
\end{tabular}

\section{PROTOCOLO M}

No grupo de Risco Básico e no grupo de Risco M édio.

\begin{tabular}{l|c|c} 
PROTOCOLO M & DOSES & DIAS / OBS \\
Mercaptina & $25 \mathrm{mg} / \mathrm{m}^{2} \mathrm{VO}$ & 1 a 56 \\
\hline MTX & $2.000 \mathrm{mg} / \mathrm{m}^{2} \mathrm{IV} \mathrm{em}$ & $8,22,36$ e 50 \\
& infusão contínua por 24 horas & (semanas pares) \\
\hline Resgate com Leucovorin & $30 \mathrm{mg} / \mathrm{m}^{2} \mathrm{NV}$ & 36 horas pós MTX \\
\cline { 2 - 3 } (conforme nível do MTX sérico) & $15 \mathrm{mg} / \mathrm{m}^{2} \mathrm{IV}$ & $48,54,60$ horas pós MTX \\
\hline MTX IT & Dose segundo a idade & $8,22,36$ e 50 \\
\hline
\end{tabular}

\section{PROTOCOLO II}

Após o Protocolo M nos grupos de Risco Básico e Risco M édio e após os Blocos HR do grupo Alto Risco:

\begin{tabular}{l|c|c} 
PROTOCOLO II & DOSES & DIAS / OBS \\
Dexametasona & $10 \mathrm{mg} / \mathrm{m}^{2} \mathrm{VO}$ & 1 a 22, com redução gradativa da dose. \\
\hline Vincristina & $1,5 \mathrm{mg} / \mathrm{m}^{2} \mathrm{IV}$ & $8,15,22$ e 29 \\
\hline Doxorubicina & $30 \mathrm{mg} / \mathrm{m}^{2} \mathrm{IV}$ & $8,15,22$ e 29 \\
\hline L-Asparaginase & $10.000 \mathrm{u} / \mathrm{m}^{2} \mathrm{IM}$ & $8,11,15$ e 18 \\
\hline Ciclofosfamida com Mesna & $1.000 \mathrm{mg} / \mathrm{m}^{2} \mathrm{IV}$ & 36 \\
\hline 6-Tioguanina & $60 \mathrm{mg} / \mathrm{m}^{2} \mathrm{VO}$ & 36 a 49 \\
\hline Citarabina & $75 \mathrm{mg} / \mathrm{m}^{2} \mathrm{IV}$ & 38 a 41 e 45 a 48 \\
\hline MTX IT & Dose segundo a idade & 38 a 45 \\
\hline
\end{tabular}

\section{BLOCO HR-1}

Após o Protocolo I no grupo Alto Risco.

\begin{tabular}{l|c|c} 
BLOCO HR-1 & DOSE & DIAS / OBS \\
MTX & $\begin{array}{c}2.000 \mathrm{mg} / \mathrm{m}^{2} \mathrm{IV} \text { em infusão contínua } \\
\text { por } 24 \text { horas (com dosagem e resgate } \\
\text { com Leucovorin conforme no Protocolo M) }\end{array}$ & 1 \\
\hline Ciclofosfamida com Mesna & $200 \mathrm{mg} / \mathrm{m}^{2} \mathrm{IV}$ & 2 a 4 \\
\hline Vincristina & $1,5 \mathrm{mg} / \mathrm{m}^{2} \mathrm{IV}$ & 1 \\
\hline Dexametasona & $20 \mathrm{mg} / \mathrm{m}^{2} \mathrm{VO}$ & 1 a 5 \\
\hline Citarabina & $2.000 \mathrm{mg} / \mathrm{m}^{2} \mathrm{IV}$ & 5 (de $12 / 12$ horas) \\
\hline MADIT & Dose segundo a idade & 1
\end{tabular}




\section{BLOCO HR-2}

\begin{tabular}{l|c|c} 
BLOCO HR-2 & DOSE & DIAS / OBS \\
Dexametasona & $20 \mathrm{mg} / \mathrm{m}^{2} \mathrm{VO}$ & $1 \mathrm{a} 5$ \\
\hline Vincristina & $1,5 \mathrm{mg} / \mathrm{m}^{2} \mathrm{IV}$ & 1 \\
\hline Daunorubicina & $30 \mathrm{mg} / \mathrm{m}^{2} \mathrm{IV}$ & 1 \\
\hline MTX & $\begin{array}{c}2.000 \mathrm{mg} / \mathrm{m}^{2} \mathrm{IV} \\
\text { em infusão contínua por } 24 \text { horas } \\
\text { (com dosagem e resgate com Leucovorin } \\
\text { conforme no Protocolo M) }\end{array}$ & 2 \\
\hline Ifosfamida com Mesna & $800 \mathrm{mg} / \mathrm{m}^{2} \mathrm{IV}$ & 6 \\
\hline Asparaginase & $10.000 \mathrm{U} / \mathrm{m}^{2} \mathrm{IM}$ & 5 \\
\hline MADIT & Dose segundo a idade & (de $12 / 12$ horas) \\
\hline
\end{tabular}

\section{BLOCO HR-3}

\begin{tabular}{l|c|c} 
BLOCO HR-3 & DOSE & DIAS / OBS \\
Dexametasona & $20 \mathrm{mg} / \mathrm{m}^{2} \mathrm{VO}$ & 1 a 5 \\
\hline Citarabina & $2.000 \mathrm{mg} / \mathrm{m}^{2} \mathrm{IV}$ & $1 \mathrm{e} \mathrm{2} \mathrm{(de} \mathrm{12/12} \mathrm{horas)}$ \\
\hline Etoposido & $100 \mathrm{mg} / \mathrm{m}^{2} \mathrm{IV}$ & 3 a 5 (de 12/12 hs - 5 doses) \\
\hline L-Asparaginase & $10.000 \mathrm{U} / \mathrm{m}^{2} \mathrm{IM}$ & 6 \\
\hline MADIT & Dose segundo a idade & 5 \\
\hline
\end{tabular}

\section{IRRADIAÇÃO CRANIANA PROFILÁTICA}

Somente na LLA-T e idade $\geq 1$ ano, com $12 \mathrm{G}$ y, após o Protocolo II do Grupo de Risco M édio e do Grupo de Risco Alto.

\section{IRRADIAÇÃO CRANIANA TERAPÊUTICA}

Pacientes com acometimento inicial do SN C recebem $18 \mathrm{~Gy}$ ( $12 \mathrm{~Gy}$ no $2^{\circ}$ ano e $0 \mathrm{~Gy}$ no $1^{\circ}$ ano de vida) após o Protocolo II, bem como duas doses adicionais de MTX IT.

\section{ACOMETIMENTO TESTICULAR INICIAL}

$\mathrm{N}$ ão está previsto orquiectomia e nem radioterapia testicular. Se após $02^{\circ}$ Bloco HR-3 permanecer dúvida quanto à normalização testicular (por meio de exame físico e ultra-sonográfico), o caso deverá ser discutido na M esa Redonda do Serviço.

N OTA: Levantamento feito no Serviço de $\mathrm{H}$ ematologia do IN CA, compreendendo o período de julho de 83 a dezembro de 98 , incluiu 109 casos de LLA, com idade que variou entre 6 meses e 15 anos (idade média 5,6 anos) e mostrou que $59(54,1 \%)$ dessas crianças eram do sexo masculino. Essas crianças foram tratadas com o Protocolo Europeu BFM/LLA- 81, 83, 86 e 90, e a grande maioria recebeu $500 \mathrm{mg} / \mathrm{m}^{2}$ de M TX em infusão contínua por 24 horas. Com um tempo de observação de 6.000 dias, gráfico de sobrevida global (SG) mediana de 3.483 dias e gráfico de sobrevida livre de doença (SLD) mediana de 2.953 dias, a SG desses pacientes foi de $63,4 \%$ e a SLD foi de $60 \%$. A literatura mostra que 0 índice de recaída testicular chegava até $8,8 \%$, previamente ao tratamento com infusão contínua de M TX. Análise feita a partir de diversos protocolos BFM (idêntico ao levantamento feito no INCA) mostrou que o grupo que não recebeu M TX em infusão contínua apresentou um índice de $6,7 \%$ de recaída testicular, enquanto que 0 que havia recebido apresentou uma taxa de recaída de 2,5\%; e 0 grupo que foi tratado com altas doses $\left(5 \mathrm{~g} / \mathrm{m}^{2}\right)$ de MTX, mostrou um índice de recaída semelhante (2,3\%). C omo a infusão do M TX contribuiu para a melhora dos resultados da LLA, a tendência agora é utilizar nas crianças com acometimento testicular 0 MTX em infusão contínua, deixando a radioterapia testicular apenas para os casos que não respondem ou para uma segunda recaida testicular. A persistência de doença local é indicativo de orquiectomia bilateral. 
Um dos objetivos desse tratamento é preservar a função gonadal, já que a radioterapia testicular leva a uma futura infertilidade.

\section{TRATAMENTO DE MANUTENÇÃO}

A duração total do tratamento de manutenção é de 24 meses (104 semanas), calculado a partir do início do Protocolo I, para todos os grupos de risco, sendo dados:

- 6-M ercaptina - $50 \mathrm{mg} / \mathrm{m}^{2}$ - Vo /dia, 2 horas após o jantar (com água).

- M TX - $20 \mathrm{mg} / \mathrm{m}^{2}$ - VO/semana.

N OTA: Crianças em $1^{\text {a }}$ recidiva ou $2^{\text {a }}$ remissão da $L L A$, crianças que não obtiveram remissão completa (que mantêm mais do que $5 \%$ de blastos na medula óssea) no D 33 da Indução e crianças com $t(9 ; 22)$ ou $t(4: 11)$ que não mostrem menos de 1.000 blastos/ $\mu$ l no sangue periférico com 7 dias de Prednisona e uma dose de MTX IT têm indicação de transplante de medula óssea alogenéico aparentado ou não aparentado.

\section{EXAMES DE ACOMPANHAMENTO}

\section{PRÉVIO AO TRATAMENTO}

- Sangue - H emograma e plaquetometria

- Sangue - Estudo da H emostasia (incluindo Fibrinogênio)

- Sangue - Glicemia

- Sangue - Avaliação da função renal com dosagem de Uréia e Creatinina

- Sangue - Eletrólitos: Sódio, Potássio, Cloretos, Cálcio e Fósforo.

- Sangue - Transferases (AST - Aspartatoamino-transferase e ALT- Alanina-aminotransferase)

- Sangue - Ácido úrico

- Sangue - D esidrogenase Lática (D H L) e Beta2 M icroglobulina

- Fezes - Parasitológico

- Eletrocardiograma

\section{AO INÍCIO DO TRATAMENTO}

- Sangue - Hemograma, Estudo da hemostasia, Glicemia, U réia, Creatinina, Ácido Ú rico, Eletrólitos, Transferases, Amilase e pesquisa sorológica para HIV,
H TLV, H epatite B e C e Sífilis.

- Ecocardiograma com Fração de Ejeção.

- Mielograma a cada mudança de fase (Protocolo I, Protocolo M, Protocolo II e $M$ anutenção) do tratamento.

- Exame citológico e bioquímico do LCR a cada punção lombar.

\section{DURANTE A FASE DE MANUTENÇÃO}

- Sangue - H emograma com plaquetometria, Transferases, Ácido Ú rico, U réia e $C$ reatinina mensalmente.

- Fezes - Parasitológico a cada 3 meses.

\section{AO TÉRMINO DO TRATAMENTO}

- Sangue - H emograma com plaquetometria, U réia, C reatinina, Ácido Ú rico, DHL e Beta2 M icroglobulina.

- M ielograma

- Exame citológico do LCR.

\section{APÓS O TÉRMINO DO TRATAMENTO}

0 paciente deverá ser avaliado a cada 2 meses no primeiro semestre, a cada 4 meses no $2^{\circ}$ semestre, a cada 6 meses no $2^{\circ}$ e $3^{\circ}$ anos e anualmente a partir do $4^{\circ}$ ano fora de tratamento, por meio de:

- Exame físico

- Exames de sangue: H emograma com plaquetometria, U réia, C reatinina, Ácido Ú rico, DHL e Beta2 M icroglobulina. As sorologias para Sífilis, Chagas, H epatite B e C, HIV e HTLV deverão ser repetidas no $24^{\circ}$ mês após a última transfusão de hemoderivado.

N OTA: A criança deverá ser acompanhada por Endocrinologista, para prevenção, avaliação e tratamento de possíveis efeitos colaterais tardios do tratamento.

\section{NOTA FINAL}

Para o IN CA, qualquer conduta aplicada em suas unidades hospitalares que se encontre fora das aqui especificadas é considerada experimental ou irregular.

\section{AGRADECIMENTO}

$\mathrm{O}$ Instituto $\mathrm{N}$ acional de $\mathrm{C}$ âncer agradece, penhoradamente, à $\mathrm{D}$ ra. N orma Wollner por sua val iosa contribuição com a revisão do texto desta Conduta do IN CA. 


\section{BIBLIOGRAFIA}

D ordelmann $M$, Reiter A, Zimmermann $M$, Fengler $R$, Henze $G$, Riehm $H$, Schrappe $M$. Intermediate dose methotrexate is as effective as high dose methotrexate in preventing isolated testicular relapse in childhood acute lymphoblastic leukemia. J Pediatr $\mathrm{H}$ ematol Oncol 1998 Sep-Oct;20(5):444-50.

Protocolo original do Estudo Terapêutico Multicêntrico ALL-BFM 95 para 0 tratamento de crianças e adolescentes com Leucemia Aguda Linfoblástica - cedido pelo Prof. H. Riehm (M edizinische $\mathrm{H}$ ochschule $\mathrm{H}$ annover) e traduzido pela D ra. Lieselotte Laun.

\section{II - LEUCEMIA MIELÓIDE AGUDA (LMA)}

\section{INTRODUÇÃO}

A Leucemia Mielóide Aguda (LMA) representa cerca de $20 \%$ das leucemias agudas na infância.

M esmo com os avanços verificados nos esquemas de tratamento e nas medidas de suporte, a expectativa de sobrevida livre de doença prolongada ainda varia de $35 \%$ a $60 \%$.

Atualmente, a associação de uma antraciclina com citarabina (ARA C) é 0 esquema terapêutico mais eficaz, observandose remissão em $75 \%$ a $80 \%$ dos casos. 0 acréscimo de outros quimioterápicos, como etoposido, tioguanina ou vincristina, tem sido utilizado por alguns grupos, porém permanece discutível se eles melhoram esses percentuais de remissão.

Apesar do elevado índice de remissão, 90\% dos pacientes apresentam recaída, caso não recebam quimioterapia de consolidação e de intensificação. A consolidação/ intensificação tem como finalidade, portanto, a erradicação de células leucêmicas residuais não detectadas pelos métodos utilizados para avaliar a remissão.
Philadelphia Chromosome-Positive $(\mathrm{Ph}+)$ Childhood Acute Lymphoblastic Leukemia: Good Initial Steroid Response Allows Early Prediction of a Favorable Treatment O utcome - M artin Schrappe et al - Blood, Vol. 92 no. 8 (0 ct 15), 1998: pp 2730-2741.

Schrappe M, Reiter A, H enze $G$, N iemeyer $C$, Bode $U$, Kuhl J, Gadner $H$, $H$ avers W, Pluss $H$, Kornhuber B, Zintl $F$, Ritter J, U rban C, Niethammer D, Riehm $H$. Prevention of CNS recurrence in childhood ALL: results with reduced radiotherapy combined with $\mathrm{CN}$ S-directed chemotherapy in four consecutive ALLBFM trials. Klin Padiatr 1998 JulAug;210(4):192-9.

Algumas estratégias são utilizadas: 0 uso de quimioterápicos que não apresentam resistência cruzada e altas doses de ARA C. Estes ciclos podem ser administrados por alguns meses, como no protocolo VAPA. Já o grupo BFM utiliza sete diferentes quimioterápicos em 6 semanas, seguido por dois cursos de ARA $C$ em altas doses.

0 tratamento de manutenção, tanto em adultos como em crianças, ainda é discutível. Em dois estudos realizados com pacientes adultos, a manutenção prolongada após quatro cursos de consolidação intensiva não melhorou a sobrevida livre de doença (SLD). Por outro lado, Bücchern e col. demonstraram que a SLD em 5 anos é de 25\% para pacientes que receberam manutenção e de apenas 13\% para os que não receberam esse tratamento. Já o estudo CCG não indica o tratamento de manutenção após consolidação e intensificação com altas doses de ARA $C$ em regime sequencial de 7 dias.

0 tratamento profilático do sistema nervoso central (SN C) nos pacientes com LM A é feito geralmente com ARA $C$ intratecal (IT) ou com ARA $C$ em altas doses, porém o grupo BFM demonstrou que a probabilidade de SLD por 5 anos é maior nos pacientes irradiados, se comparados com aqueles que não receberam radioterapia craniana. 
Com isso, o esquema de tratamento adotado no INCA é fundamentado nos protocolos do Grupo Europeu (BFM 83) e (BFM 87).

\section{CRITÉRIOS DE INCLUSÃO}

- Pacientes com diagnóstico de LM A, segundo a classificação $F A B$, idade $>12$ meses e $<16$ anos.

- Crianças com diagnóstico de LMA secundária a M ielodisplasia sem doador para transplante de medula óssea.

- Leucemia mielóide aguda secundária a quimioterapia e Leucemia Mielóide Crônica Juvenil (LMCJ).

\section{CRITÉRIOS DE EXCLUSÃO}

- Tratamento anterior com quimioterapia, exceto citorredução com $\mathrm{H}$ idroxiuréia ou ARA C por período não superior a 7 dias.

- Existência de doenças graves associadas que impeçam o uso de quimioterapia intensiva.

- LM A associada à Síndrome de D own.

- Leucemia Promielocítica.

\section{DIAGNÓSTICO E EXAMES INICIAIS}

O diagnóstico será feito segundo critérios citomorfológicos (estabelecidos na classificação $F A B$ ), citoquímicos; imunofenotípicos e de cariotipagem do aspirado de medula óssea ou do sangue periférico.

De acordo com a classificação $F A B$, distinguem-se oito subtipos morfológicos de LM A:

\begin{tabular}{|c|c|c|}
\hline Subtipo FAB & $\%$ & Critérios \\
\hline MO & 2 & $\begin{array}{l}\text { Blastos com mínima diferenciação. } \\
\text { Sudam Black e mieloperoxidase, negativos pela citoquímica. }\end{array}$ \\
\hline M1 & $10-18$ & Blastos com alguma maturação e ocasionais bastões de Aver \\
\hline M2 & $27-29$ & $\begin{array}{l}\text { Mieloblastos com diferenciação ( }<20 \% \text { de monoblastos). } \\
\text { Bastões de Auer mais proeminentes. }\end{array}$ \\
\hline M3 & $5-10$ & $\begin{array}{l}\text { Promielócitos anormais, hipergranulares com bastões de Auer } \\
\text { e as vezes núcleo reniforme ou bilobulado. }\end{array}$ \\
\hline M4 & $16-25$ & $\begin{array}{l}\text { Mieloblastos e monoblastos com diferenciação } \\
\text { M4Eo: variante que apresenta mais de } 5 \% \text { de eosinófilos displásicos. }\end{array}$ \\
\hline M5 & $13-22$ & Monoblástica; sem diferenciação, M5a ou com diferenciação, M5b. \\
\hline M6 & $1-3$ & Eritroleucemia com deseritropoese e fatores megaloblásticos. \\
\hline M7 & $4-8$ & Megacarioblástica \\
\hline
\end{tabular}

\section{EXAMES INICIAIS:}

- Mielograma com coleta de material para exames de citoquímica, imunofenotipagem, citogenética e Biologia M olecular.

- Sangue- H emograma completo e contagem de plaquetas

- Sangue - Coagulograma com dosagem do fibrinogênio

- Sangue- Avaliação hepática: ALT, AST, GamaGT, D H L, Fosfatase Alcalina, Bilirrubinas

- Sangue - G licose, U réia, Creatinina, Ácido úrico, Proteínas e frações

- Sangue - Sódio, Potássio, Cálcio, M agnésio, Cloretos, Fósforo

- Sangue - Exames sorológicos para $H$ epatite, CM V, HIV, HTLV I

- Sangue - Imunoglobulinas

- Eletrocardiograma e Ecocardiograma

- Radiografia simples de tórax (RX)

\section{EXAMES ESPECIAIS:}

Ao diagnóstico deverá ser encaminhado sangue periférico ou de medula óssea, por aspirado, para estudo de Resistência a M últiplas D rogas (MDR).

N OTA: O utros exames como ultrasonografias e exames bacteriológicos serão realizados de acordo com indicação médica.

\section{PLANO GERAL DE TRATAMENTO}

- O s pacientes serão estratificados para o tratamento segundo o Grupo de Risco.

- Todos os pacientes deverão, ao diagnóstico, ser encaminhados para realização do HLA, mesmo que a indicação para transplante alogenéico de medula óssea não seja absoluta para os pacientes do Risco Baixo.

- Os pacientes que preencham os fatores de risco do Grupo de Alto Risco serão 
considerados para Transplante Alogenéico em primeira remissão e, caso não se disponha de doador H LA compatível, deverão ser incluídos em busca de doadores não relacionados. Para estes, o congelamento de medula óssea ou precursores de sangue periférico poderá ser feito na primeira remissão, e o contato com o Centro de Transplante deverá ser feito tão logo seja obtida a remissão completa, embora o coleta deva ser realizada após a intensificação (com ARA C).

- Para os pacientes com leucemia secundária ao uso de Etoposido, o tratamento deverá ser feito sem este quimioterápico.

- 0 esquema de tratamento baseia-se nos Protocolos do grupo BFM , com as seguintes modificações:

- substituição da daunorrubicina por idarrubicina na fase de indução;

- antecipação da fase de intensificação para aqueles que não obtiveram remissão no $D$ 15 de tratamento; e

- antecipação da fase de intensificação para pacientes de Alto Risco.

\section{FATORES E GRUPOS DE RISCO}

A estratificação dos fatores de risco está baseada na análise dos resultados dos protocolos BFM 78 e 83, pelos quais foi possivel identificar um grupo de pacientes que apresenta critérios de bom prognóstico, com sobrevida livre de doença de $70 \%$.

Com base nos parâmetros e resultados dos esquemas de tratamento do grupo BFM 78 e 83 , os pacientes serão tratados de acordo com os seguintes Grupos de Risco:

- BAIXO RISCO

FAB M 1 - com bastonetes de Auer

FAB M 2 - com < de 10.000 leucócitos $/ \mathrm{mm}^{3}$

FAB M 4 - com Eosinófilos

FAB M 5 - Sem visceromegalias e com

leucometria $<20.000 / \mathrm{mm}^{3}$
Remissão no D 15 do tratamento

- ALTO RISCO

D emais apresentações

Leucemias Secundárias

Já considerando-se também os critérios citogenéticos, os grupos de risco são assim denominados:

- BAIXO RISCO

FAB M 1/M 2 com bastões de Auer et( 8;21)

FAB M 4 com eosinófilos e inv (16)

Remissão no D 15 da quimioterapia

- ALTO RISCO

Todos os demais casos

\section{CRITÉRIOS DE AVALIAÇÃO DE RESPOSTA}

- Resposta Completa: M edula óssea normocelular com $\leq 5 \%$ de blastos. Sangue periférico com $\geq 1.500$ neutrófilos/ $\mu$ l e plaquetas $\geq 100.000 / \mu l$. Ausência de qualquer sinal atribuível à LM A.

- Resposta Parcial: Medula óssea normocelular com $6 \%-25 \%$ de blastos ou acometimento orgânico pela LM A.

- Falha ou Não Resposta: Para os pacientes de Risco Baixo, demonstração de doença clínica ou medula com > de $5 \%$ de blastos ao final da consolidação. Para os demais pacientes, demonstração de doença clínica ou medula com $>$ de $5 \%$ de blastos após 0 primeiro ciclo de ARA C em altas doses.

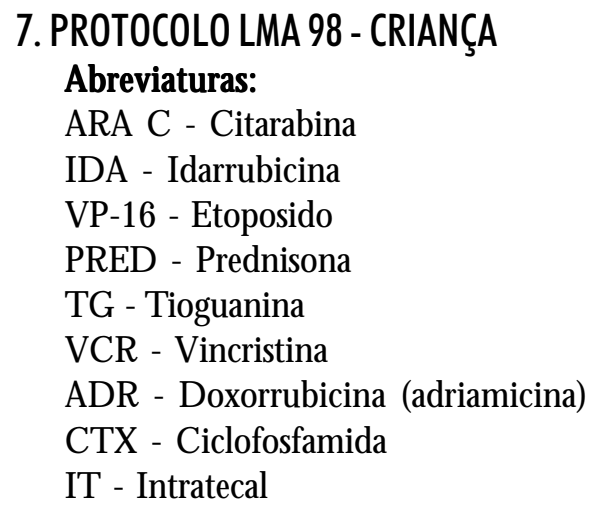

\section{PROTOCOLO LMA 98 - CRIANÇA} Abreviaturas:

ARA C - Citarabina

IDA - Idarrubicina

VP-16 - Etoposido

PRED - Prednisona

TG - Tioguanina

$V C R$ - Vincristina

ADR - D oxorrubicina (adriamicina)

IT - Intratecal

INDUÇÃO

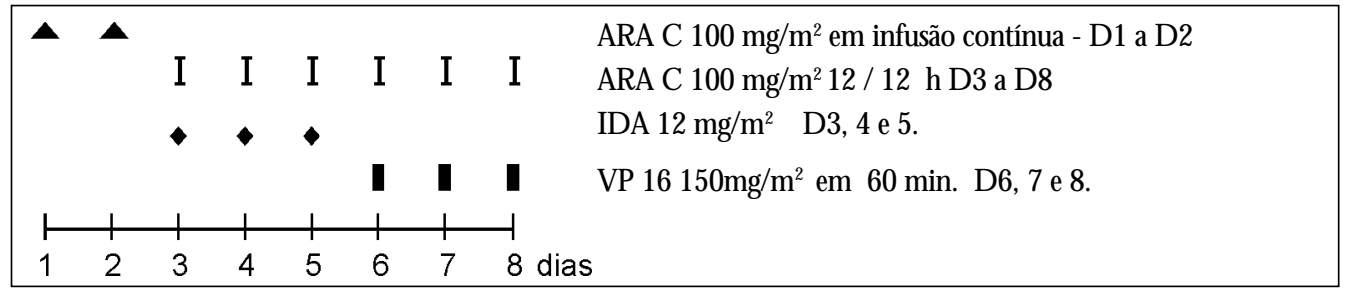




\section{CONSOLIDAÇÃO}

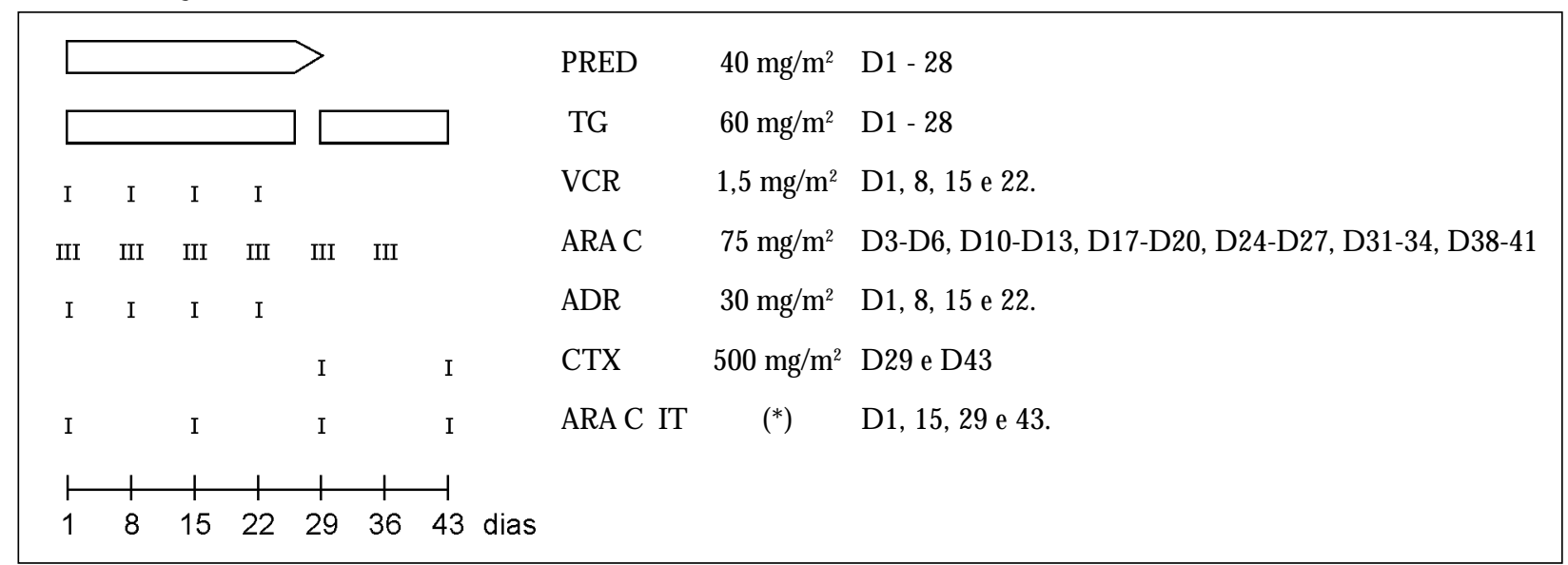

(*) D ose segundo a idade. A cada punção lombar, enviar liquor para exame citológico.

\section{ARA C/VP-16 (INTENSIFICAÇÃO)}

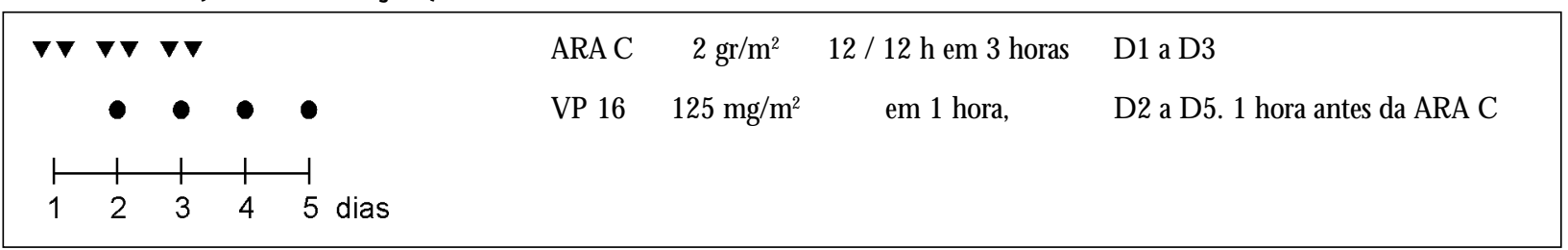

N OTA: As seguintes medidas deverão ser observadas antes do início do tratamento quimioterápico:

- Controle de processo infeccioso

- Acesso venoso profundo

- Encaminhamento de doadores de plaquetas ao Serviço de H emoterapia.

\section{MEDIDAS NA HIPERLEUCOCITOSE}

\subsection{CITORREDUÇÃO}

U tilizada para aqueles pacientes com grande massa de células leucêmicas (leucometria $>50.000 / \mathrm{mm}^{3}$ ou grandes visceromegalias), em que existe o risco de leucoestase, sangramento e síndrome de lise tumoral.

A citoredução será feita com Citarabina 50 a $100 \mathrm{mg} / \mathrm{m}^{2}$ - IV/dia. Iniciar a indução quando a leucometria atingir $20.000 / \mathrm{mm}^{3}$ ou nos casos em que, após 72 horas de quimioterapia, não se observe redução da leucometria.

Cuidadosespeciais e medidas desuporte:

- H idratação venosa: Visando a prevenir

a Síndrome de Lise Tumoral, 3.000 a
$3.500 \mathrm{ml} / \mathrm{m}^{2}$ de solução de glicose a $5 \%$, com vigilância cuidadosa do balanço hídrico e de eletrólitos.

- Alcalinização da urina com bicarbonato de sódio na hidratação.

D ose: $20 \mathrm{mEq} / 500 \mathrm{ml}$ da solução glicosada

- Alopurinol: Inicialmente usar $400 \mathrm{mg} / \mathrm{m}^{2} / \mathrm{dia}$ - VO e, após a citorredução, 200 mg/m²/dia.

- Evitar nesta fase outras medidas, exceto as estritamente necessárias, como a coleta de sangue para hemograma, eletrólitos, gasometria.

- Evitar transfusões de concentrados de hemácias, de maneira que o citócrito (hematócrito + leucócrito) permaneça $\leq 30 \%$.

- Transfundir plaquetas de acordo com o grau de plaquetopenia e risco de sangramento. D ose: $4 \mathrm{U} / \mathrm{m}^{2}$, ou preferencialmente plaquetas de doador único.

- Quimioterapia: Iniciar o mais precocemente possível.

\subsection{IRRADIAÇÃO CRANIANA}

Indicada quando houver sintomas neurológicos. Os casos serão individualmente analisados pela equipe médica do Serviço de $\mathrm{H}$ ematologia. 


\subsection{LEUCOFERESE}

Poderá ser indicada nos pacientes com sintomas associadosa hiperleucocitose, tais como sinais neurológicos ou síndrome respiratória. $N$ este caso, discutir em equipe, considerando-se as dificuldades técnicas, especialmente em crianças de baixa idade, para as quais geralmente se opta pela exsanguíneo transfusão.

\section{DETALHAMENTO DA QUIMIOTERAPIA}

\section{Indução:}

0 tratamento de indução consiste de:

- Citarabina - $100 \mathrm{mg} / \mathrm{m}^{2}$ em infusão contínua por 48 horas, iniciando no D 1

- Citarabina - $100 \mathrm{mg} / \mathrm{m}^{2}$ a cada 12 horas, infusão de 30 min. D 3 aD 8. Total de 12 doses.

- Idarubicina - 12 mg/m² infusão em 30 min. D 3 a D 5

- VP 16 - $150 \mathrm{mg} / \mathrm{m}^{2}$ em infusão de $60 \mathrm{~min}$, D6, D 7, D 8 .

- Citarabina Intratecal - D ose dependente da idade (ver a Tabela 1). Adiar este procedimento em casos de distúrbios da coagulação e plaquetopenia grave.

$\mathrm{N} 015^{\circ}$ dia de tratamento deve ser feito um aspirado de medula óssea e segundo este resultado adotar as seguintes condutas:
- A plasia e menos de 5\% de blastos- Aguardar a recuperação hematológica (neutrófilos $>1.000 / \mathrm{mm}^{3}$ e plaquetas $>50.000 / \mathrm{mm}^{3}$ ) para dar continuidade ao tratamento. Esta recuperação deverá acontecer até $029^{\circ}$ dia do tratamento. 0 mielograma deverá ser repetido para confirmar a remissão.

- M edula óssea com mais de $10 \%$ de blastos

- Prosseguir com a quimioterapia. N estes casos, se o aspirado de medula óssea mostrar-se intensamente hipocelular, é recomendado repetir o mielograma dentro de cerca de 4 dias, para confirmar a ausência de resposta completa. Q uando a contagem de blastos for duvidosa ou estiver entre $5 \%$ $10 \%$, é recomendável a repetição do mielograma para evitar equívocos, decorrentes inclusive do uso do G-CSF.

\section{Consolidação:}

U tilizada logo após a indução para os pacientes de Baixo Risco que tenham obtido a remissão no $D 15$ de tratamento. $\mathrm{N}$ os demais pacientes, esta fase será feita após as intensificações com ARA em altas doses.

\section{Fase 1 da Consolidação}

Sempre que possível esta fase deve ser feita sem pausas:

\begin{tabular}{|c|c|c|}
\hline \multicolumn{3}{|c|}{ FASE 1 DA CONSOLIDAÇÃO } \\
\hline Prednisona & $40 \mathrm{mg} / \mathrm{m}^{2}-\mathrm{VO}$ & $\begin{array}{c}\text { D1 a D28, } \\
\text { suspender em } 1 \text { semana. }\end{array}$ \\
\hline Tioguanina & $60 \mathrm{mg} / \mathrm{m}^{2}-\mathrm{VO}$ & D1 a D28 \\
\hline Vincristina & 1,5 mg/m² (máx. 2mg) & D1, 8, 15 e 22 . \\
\hline Adriamicina & $30 \mathrm{mg} / \mathrm{m}^{2}-\mathrm{IV}$ & D1, 8, 15 e 22 . \\
\hline Citarabina & $75 \mathrm{mg} / \mathrm{m}^{2}-\mathrm{IV}$ & $\begin{array}{ll}\text { D3,4,5 e } 6 . & \text { D } 10,11,12 \text { e } 13 . \\
\text { D17, 18, } 19 \text { e 20. } & \text { D24, 25, 26 e } 27 .\end{array}$ \\
\hline Citarabina intratecal & Dose segundo a idade & D1 e D15 \\
\hline
\end{tabular}

N OTA: Por ser um período dequimioterapia contínua, o uso do G-CSF só esta indicado no término da Fase 1 ou caso o paciente apresente neutropenia grave e infecção.

Fase 2 da Consolidação
Para o início da Fase 2, poderá ser feito um intervalo de no máximo 14 dias, e alguns parâmetros deverão ser observados para 0 início da ARA C e da Ciclofosfamida, quais sejam Leucócitos $>1.500 / \mathrm{m}^{2} \mathrm{~mm}^{3}$ Plaquetas $>50.000 / \mathrm{m}^{2} \mathrm{~mm}^{3}$

FASE 2 DA CONSOLIDAÇÃO

\begin{tabular}{|c|c|c|}
\hline Tioguanina & $60 \mathrm{mg} / \mathrm{m}^{2}-\mathrm{V} 0$ & D1 a D15 \\
\hline Citarabina & $75 \mathrm{mg} / \mathrm{m}^{2}-\mathrm{IV}$ & D3,4,5 e $6 . \quad$ D10, 11,12 e 13. \\
\hline Ciclofosfamida & $500 \mathrm{mg} / \mathrm{m}^{2}-\mathrm{IV}$ & D1 e D15 \\
\hline Citarabina & Dose segundo a idade & DI \\
\hline
\end{tabular}


Intensificação:

U tilizada logo após a consolidação, nos casos de pacientes de Baixo R isco que tenham obtido a remissão no D 15 de tratamento. N os demais pacientes, esta fase será antecipada para após a Indução, e consta de dois ciclos de ARA C em altas doses e VP 16.

O s seguintes critérios deverão ser observados para o início da intensificação para os pacientes de

- RISCO BAIXO:

- intervalo de pelo menos 2 semanas após a consolidação

- bom estado geral e ausência de infecção

- aspirado de medula óssea prévio

- neutrófilos $\geq 1.500 / \mathrm{mm}^{3}$ e plaquetas $\geq 100.000 / \mathrm{mm}^{3}$

Fases 1 e 2 - Intensificação (Ver adiante os cuidados para administração do ARA C).

\begin{tabular}{|c|c|c|}
\hline \multicolumn{3}{|c|}{ Fases le 2 - Intensificação } \\
\hline Citarabina & $2 \mathrm{gr} / \mathrm{m}^{2}-\mathrm{IV}-12 / 12 \mathrm{H}$ & D1 a D3. Total de 6 doses. \\
\hline VP 16 & $125 \mathrm{mg} / \mathrm{m}^{2}$ & $\begin{array}{l}\text { D2 a D5 Infusão de } 1 \text { H. } \\
\text { Iniciar } 01 \text { hora antes do ARAC }\end{array}$ \\
\hline
\end{tabular}

Pacientes de Alto Risco ecom doador H LA compatíve - D iscutir o encaminhamento para 0 transplante alogenéico após a primeira intensificação, seestiverem em RespostaC Completa.

Critérios que deverão ser observados para início da Segunda Intensificação nos pacientes de Risco Baixo:

- pausa de 1 semana após a normalização do hemograma

- estado geral bom e ausência de infecção - neutrófilos $\geq 1.500 / \mathrm{mm}^{3}$ e plaquetas $\geq 100.000 / \mathrm{mm}^{3}$.

\section{- Cuidados especiais para administração do ARA C em altas doses}

- H idratação venosa - 3.000 a $3.500 \mathrm{ml} / \mathrm{m}^{2}$ de solução de glicose a $5 \%$. Considerar a reposição de sódio e potássio.

- M anter a hidratação venosa até pelo menos 48 horas após a última dose de ARA C.

- Colírio de dexametasona - 2 gotas em cada olho de $6 / 6$ horas.

- V itamina B6 - $150 \mathrm{mg} / \mathrm{m}^{2}$ a cada 12 horas durante 7 dias.
O bservação rigorosa de surgimento de sinais neurológicos, especialmente manifestações cerebelares. C aso isto ocorra, suspender a ARA C.

\section{Profilaxia do acometimento do sistema nervoso central (SNC)}

A radioterapia craniana deve ser iniciada após a conclusão da quimioterapia intensiva.

A dose de irradiação será de acordo com a idade (ver a Tabela 1 ) e a taxa de irradiação diária deve ser de $2 \mathrm{G}$ y ao dia, ou seja $10 \mathrm{~Gy}$ por semana.

Tabela 1 - Doses profiláticas do acometimento do SNC

\begin{tabular}{lcc} 
IDADE & ARAC IT & IRRADIAÇÃO CRANIANA \\
\hline$>1-2$ anos & $26 \mathrm{mg}$ & $15 \mathrm{~Gy}$ \\
\hline$>2-3$ anos & $34 \mathrm{mg}$ & $18 \mathrm{~Gy}$ \\
\hline$>3$ anos & $40 \mathrm{mg}$ & $18 \mathrm{~Gy}$ \\
\hline
\end{tabular}

\section{Manutenção}

Consiste no uso da associação de 6 TG (Tioguanina) e ARA C por um período total de $11 / 2$ anos, a contar do primeiro dia de tratamento.

Para o início da manutenção, é obrigatório a realização de mielograma, como também pesquisa a rearranjamentos genéticos por Biologia M olecular, caso tenha sido realizada ao diagnóstico.

0 tratamento de manutenção será feito concomitante com a irradiação craniana e consiste deTG - $40 \mathrm{mg} / \mathrm{m}^{2}$ VO diário eARA C - $40 \mathrm{mg} / \mathrm{m}^{2}$ SC a cada 4 semanas por 4 dias consecutivos.

Controle do uso de TG e ARA C na Fase de Manutenção

\begin{tabular}{lcc} 
& \multicolumn{1}{c}{ Tioguanina } \\
Leucócitos & $>2.000 / \mathrm{mm}^{3}$ & $100 \%$ da dose de TG \\
\hline Leucócitos & $1.000-2.000 / \mathrm{mm}^{3}$ & $50 \%$ da dose de TG \\
\hline Leucócitos & $<1.000 / \mathrm{mm}^{3}$ & ZERO \\
\hline & \\
\hline & ARA C \\
\hline Iniciar somente se leucócitos $>2.000 / \mathrm{mm}^{3}$ e plaquetas $>80.000 / \mathrm{mm}^{3}$
\end{tabular}

\section{Tratamento do SNC}

$\mathrm{N}$ os pacientes com acometimento do SN C, manter a injeção de ARA C intratecal 
até a negativação do líquor e, após, seguir com as punções previstas no Protocolo. A irradiação craniana (Tabela 2) deverá ser feita 4 semanas após o final da quimioterapia.

\begin{tabular}{l|c}
\multicolumn{2}{c}{$\begin{array}{c}\text { Tabela } 2 \text { - Doses terapêuticas do } \\
\text { acometimento do SNC }\end{array}$} \\
\begin{tabular}{l|c} 
Idade & Dose de RT \\
\hline$>1$ - 2 anos & $24 \mathrm{~Gy}$ \\
\hline$>3$ anos & $30 \mathrm{~Gy}$ \\
\hline
\end{tabular}
\end{tabular}

\section{ORIENTAÇÕES GERAIS}

Durante o período da quimioterapia intensiva, é recomendável a seguinte vigilância:

\begin{tabular}{l|c}
\multicolumn{2}{|c}{ Indução } \\
\hline Hemograma + Plaquetas & A cada 1-2 dias \\
\hline Eletrólitos & A cada 1 - 2 dias \\
\hline Glicose & A cada 2 - 3 dias \\
\hline Coagulograma & A cada 2 - 3 dias até normalizar \\
\hline Creatinina & e, após, semanal. \\
\hline Hepatograma & A cada 2 - 3 dias \\
\hline Exames bacteriológicos & Semanal \\
\hline Ácido úrico & A critério médico \\
\hline Proteínas & Duas vezes por semana até normalizar \\
\hline
\end{tabular}

\begin{tabular}{l|c}
\multicolumn{2}{c}{ Consolidação } \\
Hemograma + Plaquetas & A cada 2 - 3 dias \\
\hline Eletrólitos & 2 - 3 vezes por semana \\
\hline Glicose & Semanal \\
\hline Coagulograma & Por indicação clínica \\
\hline Creatinina & A cada 2 - 3 dias \\
\hline Hepatograma & Semanal \\
\hline Ex. Bacteriológicos & A critério médico \\
\hline Proteínas & Semanal \\
\hline
\end{tabular}

\begin{tabular}{|c|c|}
\hline Hemograma + Plaquetas & 2 - 3 vezes por semana \\
\hline Eletrólitos & 2 - 3 vezes por semana \\
\hline Glicose & Ao iniciar a fase \\
\hline Coagulograma & Ao iniciar a fase \\
\hline Creatinina & 2 - 3 vezes por semana \\
\hline Hepatograma & Semanal \\
\hline Ex. Bacteriológicos & Quando indicado \\
\hline Proteínas & Semanal \\
\hline
\end{tabular}

\begin{tabular}{l|c}
\multicolumn{2}{c}{ Manutenção } \\
Hemograma + Plaquetas & Mensal \\
\hline Creatinina & Mensal \\
\hline Hepatograma & Mensal \\
\hline Ácido úrico & Mensal \\
\hline Desidrogenase lática & Mensal \\
\hline
\end{tabular}

\section{Ao Término do Tratamento:}

- Sangue - hemograma com plaquetometria, creatinina, hepatograma, ácido úrico e desidrogenase lática.

- M ielograma com pesquisa de rearranjos citogenéticos por Biologia M olecular, caso este tenha sido realizado ao diagnóstico.

- Exame citológico do liquor.

\section{Após o Término do Tratamento:}

0 paciente deverá ser avaliado a cada 2 meses no primeiro semestre, a cada 4 meses no $2^{\circ}$ semestre, a cada 6 meses no $2^{\circ}$ e 30 anos e anualmente a partir do $4^{\circ}$ ano fora de tratamento, por meio de:

- exame físico;

- exames de sangue: hemograma com plaquetometria, uréia, creatinina, hepatograma, ácido úrico e desidrogenase lática.

As sorologias para sífilis, hepatite $B$ e C, H IV e H TLV deverão ser repetidas no $24^{\circ}$ mês após a última transfusão de hemoderivados.

\section{Caso este protocolo de tratamento seja} aplicado a lactentes, as doses de quimioterapia deverão ser calculadas em $\mathrm{mg} / \mathrm{kg}$.

Para isto, divida a dose preconizada por 30 e multiplique pelo peso do paciente.

\section{NOTA FINAL}

Para o IN CA, qualquer conduta aplicada em suas unidades hospitalares que se encontre fora das aqui especificadas é considerada experimental ou irregular.

\section{AGRADECIMENTO}

$\mathrm{O}$ Instituto $\mathrm{N}$ acional de $\mathrm{C}$ âncer agradece, penhoradamente, à D ra. N orma Wollner por sua valiosa contribuição com a revisão do texto desta Conduta do IN CA. 


\section{BIBLIOGRAFIA}

Bishop JF, M athews JP, Young GA, et al: A randomized study of high-dose cytarabine in induction in acutemyeloid leukemia. Blood 87: 1701-1717, 1996.

Büchenner T, H iddemann W, Löfler $G$, Gassmann W, M aschmeyer G, Heit W et al. Improved cure rate by very early intensification combined with prolonged maintenance chemotherapy in patients with acute myelogenous leukemia: data from the AM L Cooperative Group. Semin H ematol 28;76-79, 1991.

Craig A H urwitz, Kevin G. Mounce, H olocombe E. Grier. Treatment of Patients with Acute M yelogenous Leukemia: Review of C linical Trials of the Past D ecade. Journal of Pediatric Hematologu O ncology 17(3): 185-197, 1995.

Creutzig U, Ritter J, Schellong G. D oes cranial irradiation reduce the risk for bone marrow relapse in acute myelogenous leukemia: unexpected results of the childhood AM L study BFM 87. Clin O ncol 11:279-286, 1993.

Creutzig U, Ritter J, Schellong G. Identification of two risk groups in childhood acute myelogenous leukemia after therapy intensification in study BFM 83 as compared with study BFM 78. Blood 75:1932, 1990.
Creutzig U. Diagnosis and treatment of acute myelogenous leukemia in childhood. Critical Reviews O ncol Hematol 1996, 22: 183-196.

$M$ andelli $F$, Vegna $M L$, Avvisati $G$, et al. A randomized study of efficacy of postremission therapy in adult acute nonlymphocytic leukemia: a report of the I talian Cooperative G roup G IM EM A. Ann Hematol 64:166-172, 1992.

Ravindranath $Y$, Steuber $C P$, K rischer J, et al. $\mathrm{H}$ igh dose cytarabine for intensification of early therapy of childhood acute myelogenous leukemia: A Pediatric O ncology Group Study. J Clin O ncol 9:572, 1991.

Weels RJ, Woods WG, Lampkin BC $N$ esbit ME, Lee JW Buckley JD, et al. Impact of high-dose cytarabine and asparaginase intensification on childhood acute myelogenous leukemia: a report from the Childrens $C$ ancer Group. J. Clin Oncol 11: 538-545, 1993.

Weinstein $H$, Mayer $R$, Rosenthal $D$, et al. Chemotherapy for acute myelogenous leukemia in children and adults. VAPA update- Blood 62:315-319, 1983.

Weinstein HJ, Ebb D - Diagnosis and treatment of childhood acute myelogenous leukemia - Ped. Clin. North America: 44(4) 847-862, 1997. 